\title{
Neue Momente in der Klassenbewegung 1973 in der Metallindustrie
}

\author{
Redak tionskollektiv Gewerkschaften*
}

\section{Vorbemerkung}

Der folgende Versuch einer Analyse der spontanen Streikbewegung in der Metallindustrie in diesem Jahr erhebt nicht den Anspruch auf Voliständigkeit. Es ging vor allem darum, die Momente herauszuarbeiten, welche die Niederlagen in diesen Kämpfen bedingen. Diese Momente werden als Schwächen der gegenwärtigen Verfassung der Gewerkschaftsorganisation entwickelt. Denn die produktive Verarbeitung dieser Niederlagen scheint uns eine wesentliche Bedingung von Lern- und Veränderungsprozessen innerhalb der Gewerkschaft zu sein. Eine vollständige Analyse müßte aber dem Anspruch gerecht werden, gleichzeitig die vorwartsweisenden Momente herauszuarbeiten. Diese werden zwar im Aufsatz kurz zu umreißsen versucht, bedürfen aber einer genaueren Analyse, die aber nur durch eine historische Behandlung der Entwicklung der Gewerkschaftsorganisation möglich scheint. In einem späteren Aufsatz soll die Frage nach den weitertreibenden Momenten der Klassenbewegung dieses Jahres dann direkt aufgenommen werden, und zwar im Zusammenhang mit der Frage nach der Entstehung und der Tragfähigkeit der gegenwärtigen Vorstellungen von Demokratisierung der Gewerkschaft, von betriebsnaher Tarifpolitik und Stärkung der Stellung der betrieblichen Vertretungsorgane (der Vertrauenskörper) innerhalb der gewerkschaftlichen Organisation. Denn da die Frage nach den weitertreibenden Momenten dieser Klassenbewegung als Frage nach den Entwicklungsansätzen der Gewerkschaft zur Klassenorganisation am Ende des Aufsatzes gestellt wird, wird es notwendig sein, die innerhalb der Gewerkschaft selbst entwickelten Konzepte, welche die notwendige Auseinandersetzung mit der Politik der IG-Metallführung eflek tieren, zu untersuchen (1).

Mit dem Lohnabschluß von $8,5 \%$ zu Beginn dieses Jahres hat die IG-Metal] gerade jene selbständige Bewegung in den Betrieben in Gang gesetzt, welche sie seit den Septemberstreiks 1969 sorgsam zu verhindern suchte. Weil sie im Winter nicht den Streik ausrief zur Erkämpfung der von den Arbeitern geforderten Lohnerhö. hungen, kämpften die Arbeiter in den einzelnen Betrieben ohne Unterstützung und ohne Legitimierung durch die Gewerkschaftsführung. Dabei hat der Kampf der Arbeiter gegen das Kapital bei gleichzeitig verstärktem Verharren ihrer Klasse norganisation, der Gewerkschaften, in der sozialpartnerschaftlichen Politik, im Vergleich

* Thomas Mitscherlich/Christel Neusüb in Diskussion mit dem Redaktionskollektiv

1) Der nachstehende Beitrag nimmt Probleme wieder auf, die wir im letzter Aufsatz, ZZum Zusammenhang von Kapitalakkumulation, Veränderungen in den Formen der Profit produktion und der Rolle neuerer Entlohnungsmethoden in der BRD" (PROKLA Nr. 4 und 5) entwickelt hatten. Er stützt sich u. a. auf Diskussionen im Rahmen von ,express"-A.rbeitskonferenzen und auf die im „express", im Gewerkschaftsspiegel, in ,Arbeiterpoli tik" veröffentlichten Berichte. 
zu den Septemberstreiks 1969 ein verändertes Gesicht bekommen.

In dem ersien Streik nach der Tarifrunde, dem Streik in einem traditionellen Avantgardebetrieb der Stahlindustrie bei Hoesch, sind schon exemplarisch die Momente enthalten, welche die Klassenbewegung in weiteren Verlauf des Jahres als neue Stufe der Entwicklung gegenüber den Septemberstreiks kennzeichnen. Während $196^{\circ}$ Betriebsrat und Vertrauensleute den Streik initiert hatten, geht er diesmal als spontane Arbeitsniederlegung von der Feineisenstrake, also von den niedrig bezahlten Schichten der Arbeiter im Betrieb aus. Die betrieblichen linken Gewerkschaftskader werden vom Streik überrascht, und da es ihnen nicht gelingt. den Streik zu organisieren, müssen sie ihn erfolglos unter den Druck der IG-Metall-Führung abbrechen. Der Streik wendet sich zum einen gegen das Spaltungsmanover der Betriebsleitung, die den Sinn der linearen Lohnerhohung durch Zuschläge zu den oberen Lohngruppen in sein gerades Gegenteil verkehrt. Zum anderen kritisiert er ummittelbar die Politik der IG-Metall: den Lohnabschlufs unterhalb der in der Urabstimmung aufgestellten Forderung und ohne breite Zustimmung der Mitglieder (2). Indem auf der Erfüllung der ursprïnglichen Forderung bestanden wird, demonstriert ein einzelner kampfstarker Betrieb seine Nichtunterwerfung unter das zentral ausgehandelte Ergebnis. Daß der Streik scheitert, liegt wohl nicht zum wenigsten daran, dak er, im Unterschied zu 1969, keine Bewegung in anderen Betrieben auslöst. Aber nicht nur das. Schon einen Tag nach Beginn des Streiks 1969 bei Hoesch beschließt die Verhandlungskomimission der IG-Metall, die Tarifverhandlungen für die Stahlindustrie vorfristig einzuleiten. Demgegenüber verurteilt Eugen Loderer den Streik 1973 mit dem Argument, dic Hüttenarbeiter hätten .,sich und der Gewerkschaft durch ihre Aktionen Schaden zugefügt" (3).

Fassen wir die neuen Momente, welche im Hinblick auf die spätere Streikbewegung hier zuerst sichtbar werden, zusammen:

1. Im Unterschied zu 1969 kommt es 1973 nicht zu einer geschlossenen Wetle von Streiks. Der Hoesch-Streik unmittelbar nach dem Tarifabschluß hat keine auslösende Funktion.

2 Der Widerspruch zwischen der Politik der IG-Metall-Führung und ihren Mitgliedem arbeitet sich schärfer heraus. Die Kampfbereitschaft der Arbeiter wird nicht mehr in eine Machtposition der Gewerkschaft bei den Verhandlungen mit dem Kapital umgesetzt.

3. Im Streik zeigen sich Grenzen der gegenwärtigen Politik der linken betrieblichen Gewerkschaftskader.

4. Die Politik der Gewerkschaftsführung und die Schwäche einzelbetrieblicher Kämpfe wird vom Kapital unmittelbar in die Stärkung der eigenen Machtposition, in Zersplitterung und in Niederlagen der Streikbewegung umgesetzt. Das Kapital hat seit $1969 \mathrm{im}$ bewußten Klassenkampf von oben außerordnetlich gelernt.

5. Die Streikinitiative verlagert sich auf die unteren Schichten der Lohnarbeiter.

2) In der Urabstimmung spachen sich $66.6 \%$ der abstimntenden Arbeiter gegen das Ergebnis aus. In Einzelberricben wie bei Hossch war die Abstimmungsniederlage der IG-Metall-1ührung noch weit höhı̀r.

3) Gewerkschaftsspicget Nr. 5/1973, S. 11 und 13 
Die Inhalte der Streikbewegung nehmen, wie noch zu zeigen sein wird, cinen neuen Charakter an.

Im folgenden soll versucht werden, diese Momente, wenn auch nicht der Reihe nach, genauer zu entfalten, um die Punkte kennzeichnen und als Fragen herausarbeiten zu können, die die Schwäche der gegenwärtigen Gewerkschaftsbewegung in der BRD ausmachen. Denn um eine Schwäche handelt es sich in der Tat: Trotz der scheinbar günstigen konjunkturellen Situation sind die Kämpfe gerade in den Groksbetrieben länger, härter und erfolgloser als 1969 (4). Die Unnachgiebigkeit des Kapitals gegenüber Kämpfen und deren Zersplitterung führt zu Niederlagen z. B. bei Hoesch und Klöckner, zu verschwindend geringen Ergebnissen z. B. bei Rheinstahl und Küppersbuch (AEG). Wo das Kapital einen Spielraum zur politischen Strategie hat, nutzt es ihn aus, um den Arbeitern durch Niederlagen die Nutllosigkeit und Ohnmacht von Kämpfen einzubleuen. Nur dort erzielen Streikbewegungen vergleichsweise schnelle Erfolge, wo Klein- und Mittelbetriphe aufgrund hrer ök onomischen Situation (z. B. Lieferbedingungen, gute ,Ertragslage') zu Zugeständnissen gezwungen werden können. Wurde in den Septemberstreiks schon nach kurzer Streikdauer den Forderungen in den meisten Fällen nachgegeben (5), konnte die Gewerkschaft zudem die unmittelbar während der Streikbewegung vorzeitig aufgenommenen Tarifverhandlungen erfolgreich zuendeführen, so stellt sich 1973 ein weitgehend verändertes Bild dar.

4) Den Zusammenhang zwischen der Entwieklung des Kapitals und der Lage der Arbeiterklasse im Gang der Akkumulation haben wir im Aufatz ,Zum Zusammenhang von Kapitalakkumulation..." a. a. O., bis hin zu den neuerdings sich entwickelnden langfristig stagnativen Tendenzen der Akkumulation zu entwickeln versucht. Fine genauere A nalyse der gegenwartigen Situation müste untersuchen, wieweit die von Semmler/loffmann in: Kapitalakkumulation, Staatseingriffe und Lohnbewegung, PROKLA Nr. 2, theoretisch entwickelten Momente der Form widersprïchlicher Kapitalakkumulation, wo diese selbst nur noch auf der Grundlage von Stagnation bzw. Senkung des Lebensniveaus der Arbciterklasse und progressiv sich entwickelnder Arbeitslosigkcit statfindet, sich heute herauszuentwicksln beginnen.

5) IMSF (Hrig.), Die Septemberstreiks 1969, Frankfurt a. M. 1969. „Allgemein ist infolge der konjunkturellen Entwicklung der okonomische Spielraum der Konzerne gröker geworden. Deshalb besteht in einer unmittelbaren Kampfsituation die Neigung, relativ schnell zu matcricllen Zugeständnissen bereit zu sein." (Lbd., S. 358) 


\section{Die Streikbewegung wird zersplittert}

Die Bedingungen, die die Kämpfe 1969 als spontane, nicht gewerkschafulich organisierte, entscheidend hervorgebracht hatten, resultierten aus Tarifverträgen, welche am Ende der Krise mit aukerordentlich langen Laufzeiten und nur niedrigen Lohnerhöhungen abgeschlossen worden waren (6). Es kam 1969 zu einer geschlossenen Welle von Streiks, welche in gewerkschaftliche Tarifverhandlungen mündete. Zum ersten Mal wurde von den Arbeitern die Erfahrung der eigenen Stärke und eines Aktionsspielraums ohne die Gewerkschaft gemacht. Die Kämpfe selbst bildeten so crst die Grundlage dafür, dak sich in den Betrieben ein Bewußtsein von der Möglichkeit solcher Kampfformen herausbildete. Doch schienen sie in den nächsten Jahren zunächst nicht weiter notwendig. Die Abschliusse 1970 (zwischen $10 \%$ und 12,9\%) wurden als mit der Gewerkschaft erkämpfte Erfolge aufgefakt, und auch der Tarifkampf 1971 wurde noch als wenn auch weniger erfolgreicher, so doch als gewerkschaftlicher Kampf begriffen (Streik in Nordwürttemberg - Nordbaden Ende 1971). (7) Angesichts der Laufzeit von 13 Monaten und der rapide ansteigenden Inflationsraten hatten große Teile der Arbeiter für 1973 einen gewerkschaftlich organisierten Streik erwartet. So konnte der Tarifabschluk von 8,5\% Anfang $1973 \mathrm{im} \mathrm{Gegensatz}$ zu früheren Abschlïssen von seiten der streikbereiten Arbeiter und auch der betrieblichen Gewerkschaftskader nur als ein bewußtes Hintergehen der eigenen Interessen durch die Gewerkschaftsführung wahrgenonmen werden.

Doch die allgemein besonders im Ruhrgebiet erwartete und $z$. T. erhoffte, für den Sommer bzw. Herbst prognostizierte neue und mächtige Streikwelle trat in dieser Form nicht ein. Zunächst gelang es dem Kapital, die Stahlindustrie von der metallverarbeitenden Industrie abzuspalten. War 1969 ausschließlich im Stahlbereich gek̈̈mpft worden, so wurde es für die Unternehmer direkt nach dem Tarifabschluls 1973 notwendig, die ,traditionelle* Kampferfahrung der Arbeiter in den Stahlbetrieben für eine allgemeine Streikben egung unwirksam zu machen. So wurden Befriedungsversuche in den ,traditionellen Vorreiterbetrieben direkt nach dem Abschluh 1973 durch Sonderzulagen unternommen. Die Widersprüchlichkeit der gewerkschaftlichen Tarifpolitik wird während dieser Zeit in der Beurteilung der berrieblichen Zulagen durch Loderer deutich: er spricht „vom Wildwuchs in den Betrieben" und betont gleichzeitig, dath die IG-Metal! bei dem Kapital nicht um neue Verhandlungen nachsuchen wird. Die Kritik an dem "Betriebssyndikalismus" hatte sich traditionell gegen rechte Betriebsräte gerichted, die durch Betriebsvereinbarungen über höhere Effektivlöhne die gewerkschaftliche Tarifpolitik unterliefen, indem die Belegschaften von kapitalkräfigen Betrieben aus der Tarifbewegung vorzeitig herausgebrochen worden waren. Doch in diesem Fall richtete sich die Kritik von Loderer nicht gegen die Betriebsrätc, sondern gegen die Vertrauensleute, wie etwa bei Hoesch, die nicht bereit waren, die Interessen der Arbeiter zu vernachläs-

6) Der Tarifvertrag vom 1.1. 1967 bis zum 1.4.1968 erbrachte eine Erhöhung von $1.9 \%$. Der Tarifvertrag. der für die Dauer vom 1. 4.1968 bis 31.12.1970 vorgesehen war, crbrachte cine Lohnerhöhng für 1968 von $4 \%$ für 1969 von $3 \%$.

7) Bei Hoesch war es damals schon zu einer Protestdemonstration gegen den uberhasteten Abschlub durch die IG-Metall in der Stahlindustric gekommen. 
sigen. Der Abschluß von 8,5\% hatte bewußt der ,betriebssyndikalistischen " Tendenz Vorschub geleistet; die reale Einkommenssteigerung in der Metallinubive - nach Angaben der Kapitalseite lag bereits im ersten Quartal 1973 mit winer $11 \%$ über der gewerkschaftlichen Forderung. Loderer spricht hier nicht von cincm „Vertragsbruch" durch das Kapital, das ohne Kündigung der Tarifveriräge Zulagen bezahlt, oft ohne Forderung der Belegschaften oder des Betriebsrates besonders an jene, die in den Jahren zuvor die Kämpfe entfacht hatten, also an die Facharbeiter. Mit einer solchen Argumentation hätte er eine Forderung nach Neucrônung der Tarifverhandlungen begründen können (8). Nachdem seit April des Jahres aus einer Fülle von Betrieben und Ortsverwaltungen Forderungen der Vertreterversammlungen und betrieblichen Vertrauenskörper nach Entfristung der Tarifverträge, nach zusätzlichen Tarifvereinbarungen, nach gewerkschaftlichen Verhandlungen zum Ausgleich des Preisanstiegs an die IG-Metall-Leitung herangetragen worden waren, finden im Mai Spitzengespräche mit Gesamtmetall statt. In ihnen wird der Stahlindustrie eine Teuerungszulage von 280 DM zugestanden, nicht aber den Betricben der metallverarbeitenden Industrie (9). Dies, obwohl die Streikbewegung in den Einzelbetrieben schon begonnen hat und trotz der sicheren Erwartung weiterer Streiks. Auch wenn hier unterschiedliche Profitbedingungen in den einzelnen Branchen und zwischen den Betrieben angenommen werden, so ist andererseits doch klar, daß Gesamtmetall bewußt die Strategie verfolgt, die kampfstarken Betriebe der stahlverarbeitenden Industrie von einer erwarteten Streikbewegung in der gesamten Metallindustrie abzuschneiden; d. h. hier wird schon die Strategie "Eine Lektion erteilen" geplant.

Die Wahrscheinlichkeit einer Streikinitiative verschob sich damit auf die metallverarbeitende Industrie, also auf eine viel stärker von Mittelbetrieben bestimmte Branche und auf Belegschaften mit wesentlich geringerer Kampferfahrung und geringer entwickelten betrieblichen Kadern. Diese Struktur der metallverarbeitenden Industrie erschwert die spontane Vereinheitlichung einer Streikbewegung.

So kommt es z. B. in Nordbaden/Nordwürttemberg schon in der ersten $\mathbb{J}$ ahreshälfte zu den meisten Streiks. 1969 hatten dort keine Streiks stattgefunden. Im ersten Quartal 1973 waren hier die meisten betrieblichen Zulagen schon bezahit

8) Im späteren Verlauf wird das A rgument des Wildwuchses direkt gegen die akliven Ver trauensleute verwendet, indem gesagt wird, als I unktionäre der IG-Metall seien sic verpflichtet, sich an die Tarifvertrage zu halten und die Tarifautonomic nicht zu unterlaufen, da ansonsten die Organisation regrefspflichtig gemacht werden könnte. Betricbsrate stimmen mit dieser Argumentation der Matsregelung und der Entlassung in etlichen Fällen zu.

9) Dic Einschätzung Loderers, in der metallverarbeitenden Industrie seicn dic Ergebnissc der Tarifrunde mit Befriedigung aufgenommen worden, mag wohl zu diesem Lrgebni beigetragen haben, erwies sich aber offensichtlich als völlig falsch. Ubrigens wurden auch die Ergebnisse dieser Spitzenverhandlungen, aber auch ihre form (nicht einmal Hinzuziehung der Tarifkommission) durch den Streik bei Mannesmann/Huckingen und bei Rheinstahl/Duisburg kritisicrt. Zudem kamen die massenhaften Forderungen nach Entfristung der Tarifverträge, nach einer gewerkschaftlichen Vereinbarung einer Teucrungszulage cte. sowohl von den Vertrauensleuten der Stahl-als auch der metallverarbeitenden Industric. Die Forderungen wurden von Mirte April bis Mitte Mai an dic Ciewerkschaftsleitung gerichtet (vgl. dazu Berichterstattung im , express" und im , (Gewerkschaftsspicgel"). 
worden. Bei Daimler-Benz bekamen die Facharbeiter unmittelbar nach dem Tarifabschlul bis $\angle u \quad 6 \%$ zusätzliche Lohnerhöhungen, ausgehandelt vom rechten Betriebsrat. Nachdem in dem einen Betrieb eine Zulage gewährt wurde, begannen die Belegschaften in Nachbarbetrieben sich Zulagen zu erkämpten. Als die Verhandlungen um cine Teuerungszulage auf Bundesebene für die metallverarbeitende Industrie kein Ergebnis brachten (10), häuften sich die Streiks. Dabei kam es aber nur in Mamneim u einer Welle, wo in 22 Betrieben zur gleichen Zeit gestreikt wurde, wobei hier betriebliche und ortliche Gewerkschaftsfunktionäre den Kampf um Zulagen unterstützten, um die Gewerkschaft zu einer tariflichen Absicherung der Zulagen zu zwingen. (Übrigens war Daimler-Benz wiederum durch eine emeute Zulage als potentieller Streik betrieb herausgebrochen worden.) Damit war die Bewegung in Nordbaden/Nordwürt temberg ausgelaufen.

Der Anfang der zweiten Jahreshälfte war gekennzeichnet durch eine große Anzahl von Streiks in den verschiedensten Teilen der BRD. Um nur einige zu nemnen: VW/Kassel, Karmann/Osnabruck, Vulkanwerft/Bremen, Klocknerhutte/. Bremen, Hella/Lippstadt. Diese Kämpfe bildeten jedoch keine einheitliche Streikwelle. Bei dem Avantgardebetrieb Klöckner kommt es zu einer Niederlage (11). So hat das Kapital die Moglichkeit, streikende Belegschaften hinzuhalten oder offensiv gegen sie vorzugehen. Indem der Juli keine geschlossene Streikbewegung brachte, verstärkte sich in Einzelbetrieben die Tendenz, ohne eigene Aktion durch den Betriebsrat Zulagen aushandeh zu lassen, selbst wenn sic einem nicht ausreichend erschienen. Nach den Sommerferien kam es dann in Nordrheinwestfalen nach fast eineinhalb Wochen Streik bei Pierburg/Neuss und nach fün Tagen Streik bei Küppersbusch/Gelsenkirchen durch den Streik bei Opel (12) in über 40 Betrieben zum Streik, wobei jedoch in der gleichen Zeit in weit über 200 Betrieben ohne Aktionen Teuerungszulagen ausgehandelt werden (13). Das Kapital konnte so die

10) Die Verhandungen wurden entgegen dei lordermgen un Teurungszalage aus den

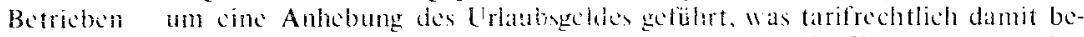
gründet wurde, dats so das Kapital im näbisten latu anch noch die linhöhungen zahlen müssic

11) Der Klocknerstreik im Juni ist ihnlich wis der Hocsehsterk zu sehen. Her kam es kurz nach der tartichen Zulage von $280 \mathrm{DM} 2 \mathrm{z}$ dem Kampt, nachdem die Arbeiter in der Vulkanwerfi in Brencen cinen lerfole crrcieht hat tels. Dabei versuchte das Internehmen de Schwachen im Verhalthis zwischen Botriebsrat und Belegsehalten auszunutzen. Der SPD-Arbeitsdircktor war wesentlich daran betciligt. Bei Klockner hatte sich in der Bekgsehaft gegenüber dem Betriebsrat das Verstandnis horansebildet, sich auf ihre starke Interessenvertretung auch in der aktiven Organisierung des Strciks ein Stück weit verlassen zu können und von daher sclbst nicht ummitleibar aktiv an der Durchführung des Streiks teilzunchmen. So war cincrscit dis Lontifikation mit den Streikmahnahmen unterentwickelt, andererseits boten die tehlende anmittelbare Kommunikation und Entschejdungsfahigkeit der Streikenden der Enternehmensleitung đis Möglichkeit, durch Tricks (scheinbare Wiederaufnahne der Arbeit) und Drohungen die Arbeiter zu verwirren (vgl. genatuer: .Arbeiterpolitik", 14. Jg., Nr. 4, Oktuber 1973 ).

12) Exemplarisch für das Verhalten des Kapitals in der Automobilindustric ist dats bei Opel gestreikt wird, wahrend bei $V W$ und BMW unverzüglich Zulagen gewihrt werden.

13) Dazu noch zwei Beispicle: bei küppersbusch in Gelsenkirchen ist die AEG erst nach fünf Tagen zu einem Zugeständnis bereit: $180 \mathrm{DM}$. Einige Wochen später werden im gesamten Konzern 200 DM gew:ihrt, so dats die Arbeiter bei Küuppersbusch weitere 20 DM ohne 
Widerspriche in der Bewegung als auch die Widersprüche in der gewerkschaftlichen Tarifpolitik ausnützen. Die Auseinandersetzung bei Ford'Köln. die mit einem organisatorischen Desaster endet, ist dabei die offensichtlichste Erscheinung (14).

Kampt erhalten. Bei Opel geschieht ahnliches: nach dem Streik werden 180 D.11 bezahlt (plus einem Vorgriff von $100 \mathrm{DM}$ aut das Weihnachtsgeld) urld rach eingen Tagen werden weitere 100 D.t zugestanden, zu einem Zeitpunkt also. als dieser zusarzliche Betrag nicht mehr in der Öfenthchkeit und damit in den anderen Berrieben diskutier uerden kann.

14) Die beiden grofien Struk bei Opel/Bochum und Ford koln zeigen dabei im Verhalten der IG-Metall sehr ahnliche Monente. Bei Opel sind de unteren Schichten der Arbeiter noth zum gröberen Teil deutche Kollegen: aufgrund der Firise im Bergbau. Der Betriebsratsvorsitzende führt her gezwungenermaben die Verhandungen. wobet er die Streikenden zugleich in der öffentlichksit diffamiert. Die Verhandlungen werden sofort auf Konzernebene getührt. "odurch wie der kontrolle durch die Streikenden entzogen sind und am Ende die Belegschatten des Konzerns (Bochum Russelsheim Kaiserslautern gegeneinander attge spielt werden und der Streik so auf Bochum reduziert bleibt. Nach drej Tagen kommt w zu einem Ergubns, das von den Streikenden abgelehnt wird, doch dic Belegschat hat nun koin Organ. das verhandeln kann, sie bertrauten auf dic lertrauensleute und einige aktive Betriebsäte. die jedoch im Betriebsrat in der Minderheit sind. Betricbsrat und Geschäftsleitung srzwingen jetzt die Aufnahme der Arbeit. Der Betriebrratsvorsitzende spricht davon, dat, de Streikenden seine Verhandlungen stören wurden und schuchtert dic Vertrauensleute mit dem Argument cin, der Streik seigewerkschaftsschädigend. Der Streik wurde so am vierten Tag abgebrochen. Bei Ford operieren der Betricberat und die Geschaftsleitung in ahnlicher Weise. Wo bei Opel die Streikfront nicht offensichtlich auseinanderbricht, zerfäll sie hier shr schnell in zwei Teile. Wah. rend bei Opel die Arbeiter im Werk blieben und die Lntemehmensleitung hiergegen keinen Angritf durchtührt. um dic Streikschiehten nicht bezahlen zu müssen. schlietst Ford das Werk, um den Streik zu spalten. Die Organisation greift dagegen nicht ein. sondern man hofft aut ein Totaufen der Bewegung. Die Halle, von der die Beweglang ausgegangen war. bildete sofort eine Streikleitung. die sich jedoch während des Kamptes entscheidend änderte. Der Betriebsratsvorsitzende verlagerte die Verhandlungen bew ubt von der Werksebene auf dic Konzernebene. un sich der Kontrolle zu entziehen. Die Forderungen lehnte er als ..utopisch" a b. Damit war der Vertrauensleutekörper gespalten. Die Streiklejtung kampt te um die Anerkennung durch den Betriebsrat und Legalisierung des Streiks durch diesen. Hierdurch entfremden sich Betriebsrat und Streiklejtung. wic auch die ausländischen und deutsehen Kollegen. Durch die Werksichliefoung wird der Streik jęzt fast ausschlicklich durch die türkischen Arbeiter getragen. (Die Forderung nach der Legalisierung des Streiks resulticrt aus der Yoglichleit in der Türkei. die der Gewerkschaft nachträglich die Legalisierung des Streiks erlatibt.) Die deutuchen Arbeiter. die nicht in der Streikleitung adiquat vertreten sind. orientieren sich an dem Betriebsrat. Die Vertrattensleute, in der Mehrzahl deutsche kollegen, bleiben inaktiv. Ihre einzige Aktivität, am vierten Streiktag eine Demonstratien zu organisieren, um wjeder die Initiative zu crlangen, wird von dem Betriebsratsvorsitzenden hintertrieben. indem er ihnen verschweigt, dats auch an diesem Tag das Werk geschlossen bleibt. Die Vertrauensleute sehen andererseits nicht ihre Aufgabe in der direkten Auseinandersetzung - es kommt nicht einmal zu einer Vertrauensleuteversammlung - sondern orientieren sich mehr an der Aufgabe, die betricbsnahe Tarifpolitik in der Organisation durchzusetzen. Auch bei ihnen hat das Argument verfangen, dafs sie alc Funktionäre an die Friedensplicht gebunden sind. Dic Streikleitung begeht dann einen weiteren schweren Fehler. indem sie das Angebot, zusammen mit dem Betricbsrat und der Geschäftsieitung zu verhandeln, ablehnt. Die letzte Möglichkeit. die deutschen und ausländischen Kollegen wieder zu einer Aktionseinheit zuruickzutühren. war damit verloren gegangen. So kann, wie bei Opel, die Geschaftsleitung und der Betriebsrat nach dem Verhandlungsergebnis hier die Arbeitsaufnahme gewaltsam erzwingen. 
Wahrend dieser Streikwelle kündigen IG-Metall und Gesamtmetall ein Spitzengespräch an. Doch durch die Zersplitterung und Spaltung der Klassenbewegung durch das Kapital. die durch die Politik der IG-Metall moglich wurde. versanden diese Verhandlungen $z w a n g s l a ̈ u f i g$, da die Bewegung. die von den Betrieben ausging. letztlich gebrochen war, da kein über den Einzelbetrieb übergreifendes Moment für die Vertranensleute und Belegschaften ersichtlich war.

Ein die Strategie des Kapitals kennzeichnender Punkt scheint uns die Tatsache zu sein. dass es in den Verhandlungen im Mai der Gewerkschaftsführung keine Zugestandrisse in Bezug auf die metallverarbeitende Industrie macht. Damit nutzt das Kapital die mangelnde Bereitschaft der IG-Mletallfuhrung, die beginnenden Streiks zu unterstützen und in eine Verhandlungsposition gegenüber den Kapital umzusetzen, zur Zersplitterung der Streikbewegung. Es gesteht damit aber auch der Gewerkschaft keine durch Zugeständnisse erreichte Integrationsfunktion mehr zu. sondern treibt sie in eine Position, wo sie sich den Arbeitern gegenüber vor allem als Disziplinierungsinstrument darstellt. Die auf betrieblicher Ebene mit den Betriebsräten ausgehandelten Zugeständnisse sind das weitere Moment der Zersplitterung der Lohnbewegung. Sie stärken zudem die Position unternehmensfreundlicher Betriebsräte und wirken damit auch wieder in Richtung einer Schwächung der Gewerkschaft als potentiell vereinheitlichender Organisation der Arbeiter.

Dabei hatte sich allerdings die Gewerkschaftsführung von vornherein in eine Situation gebracht. wo die von ihr beanspruchte Repräsentation der Gewerkschaftseinheit nur noch als von ihren Inhalten verselbständigte Form propagiert werden konnte: Gewerkschaftseinheit nicht als Machtpotenz der Arbeiterklasse gegenüber dem Kapital zur Sicherung ihrer Reproduktionsbedingungen. sondern als Machtpotenz der bürgerlichen Gesellschaft zur Festlegung der Arbeiter auf eine das Kapitalwachstum und die .Stabilität des Gesamtsystems begünstigende Forderungspolitik. Der Versuch der Gewerkschaft, den aus den Betrieben kommenden Forderungen für die Tarifrunde 1973 eine lohnpolitische Stillhaltepolitik entgegenzustellen und sie ohne Rücksicht auf die Kampfbereitschaft der Arbeiter durchzusetzen, entspricht auf der anderen Seite die Unfähigkeit, den betriebsegoistischen Tendenzen, wie sie gerade von rechten Betriebsräten repräsentiert werden, entgegenzutreten. Auf der 23. Ordentlichen Bezirkskonferenz der IG-Metall in Essen weist Loderer darauf hin, .dak sich die IG-Metall, gegen ein Auseinanderklaffen von tariflichen und betrieblichen Entlohnungsbedingungen* wehren müsse. Die Tätigkeit der Betriebsräte darf sich nicht von der Organisation entfernen, sondern müsse in ein schlüssiges Konzept gewerkschaftlicher Betriebspolitik eingebettet werden. 'Gegen unberechtigte Kritik, gegen Alleingänge von Funktionären müsse die Gewerkschaft offensiv vorgehen" (15). Über den Hoesch-Streik berichtet der Gewerkschaftsspiegel: „Eugen Loderer ... nimmt eine ablehnende Haltung zu den Aktionen der Hoesch-Arbeiter ein. Er erklärt, die Hüttenarbeiter hätten , sich und den Gewerkschaften durch ihre Aktionen Schaden zugefügt ${ }^{\circ}$. Die Streikenden haben für diese Haltung kein Verständnis und kontern mit der Frage, ob es nicht vielmehr unzureichende Tarifabschlüse seien, die den Gewerkschaften Schaden zufügten" (16). In anderem Zusam-

15) Ruhr-Nachrichten, Zeitung für Getsenkirchen. Nr. 98

16) Gewerkschaftsspiegel, Nr. 5/1973, S. 13 
menhang wiederum warnt Loderer vor dem ..innerbetrieblichen Wildwuchs" bei der Entlohnung. Indem die Politik der Gewerkschaftsführung sich nach den Stabilitats. zielen der SPD richtet, gibt sie dem Kapital freien Raum. die Gewerkschaftsorganisation durch Zugeständnisse auf betrieblicher Ebene zu schwächen, gibt sie dem Kapital die Möglichkeit, Kämpfe in Niederlagen umzuwandeln. damit aber selbst wieder die Verhandlungsposition der Gewerkschaft für Tarifverhandiungen zu un. tergraben.

Die Widersprüche zwischen der Politik der IG-Metall-Fühung und den Arbeirerinteressen kommen klarer zum Ausdruck

Nachdem wir versucht haben. die Niederlagen und die Zersplitterung der Streikbewegung aus der bewufsten, die Polıtik der Gewerkschaftsführung ausnützenden Strategie des Kapitals zu begründen. solles im folgenden darum gehen. die neuen lnhalte der Streikbewegung als Grundlage des 1973 klar hervortretenden Widerspruchs zwischen Gewerkschaftspolitik und Arbeiterinteressen darzustellen.

Nachdem die IG-Metall darauf verzichtet hatte, den bestehenden Spielraum für Lohnerhöhungen auszunutzen im Glauben nämlich, sie könne als Verwalter des Preises der Ware Arbeitskraft der SPD-Regierung einen niedrigen Preis als Siabilitätsopfer präsentieren. ohne Rucksicht auf die Lage und Kampfbereitschaft der Arbeiter und die Konjunktur des Kapitals, da wird ihr sowohl vom Kapital (durch betriebliche Lohnzugeständnisse), als auch von den kämpfenden Arbeitern vorexerziert, daß sie nicht in der Lage ist, den Preis der Ware Arbeitskraft als gesamtwirtschaftliche Größe zu planen. Die Fiktion der Gewerkschaftsführung, den Lohn als gesamtwirtschaftliche Einkommens- und Verteilungskategorie politisch verwalren und damit sich selbst zum Instrument staatlicher Wirtschaftspolitik machen zu konnen, schlägt sich in zunehmendem Widerspruch zwischen Gewerkschaftstuhrung und Teilen der Arbeiterklasse nieder.

Daß der Lohn Ausgangspunkt des Klassenwiderspruchs und der Klassenauseinandersetzung ist und nicht einfach eine makrookonomisch, durch zentrale Tarifverhandlungen zu manipulierende Rechengröße unter anderen, darauf wird die Gewerkschaft zunehmend auch durch den Inhalt der Kämpfe verwiesen. Schon im Hoesch-Streik wird durch das Pochen auf die lineare Lohnerhöhung sichtbar, dals die Arbeiter ihre Forderungen gegenüber dem Kapital an den Bedürfnissen der Reproduktion der Arbeitskraft, an ihren eigenen Lebensbedürfnissen orientieren. Die Arbeiter berufen sich hier nicht mehr auf tradierte Vorstellungen leistungsge. rechter ${ }^{`}$ Entlohnung. Nicht zufällig fält die Distanzierung der IG-Metallführung vom Hoesch-Streik mit ihrer Kritik an den betrieblichen Gewerkschaftern zusammen, welche die lineare Lohnforderung in die Tarifrunde eingebracht hatten. Und in der Tat ist die Linie der Gewerkschaftsführung, den Lohn als gesamtwirtschaftliche Einkommenskategorie verwalten zu wollen, nur auf der Basis der Vorstellung zu begründen, im Lohn erhalte der Produktionsfaktor Arbeit seinen, gerechten'Anteil an dem von ,den Produktionsfaktoren Arbeit, Boden und Kapital ' gemeinsam produzierten Produkt. Wenn demgegenüber die Klassenbewegung des Jahres 1973, aus- 
gehend ron der Verreidigung der linearen Lohnerhöhung bei Hoesch, weitergeführt durch die Kämpfe un die Ausdehnung des Crlaubs für auslandische Arhelter bei Karmann Osnabrick. verallgemeinert über die Kämpte um Teuentugszulagis. seinen Hohepunkt findend im Kampf vor allem der turkischen Arbeiter bei Ford um 1 DM fir alle. Verbesserung der A beitsbedingungen. Verminderung der BandgeschwindigWeit. 6 Wochen Crlaub. Rucknahme der Entlassungen. Wenn sich so die Klassenbewegung inhaltich auf die Reproduktionsbeingungen der Arbeitskraft bezieht dieso gegenuber dem Kapital enfordert: dann inuti de an Einkonmensgrolven im Rahnen kapitalistischer Wirtschaftspolitik orientierte. in diesem Sinn also burgerliche Politik der Gewerkschafssührnng sich in Widerspruch zum Klasseninhalt der okonomischen' Kanpte setzen.

Konnten die Septemberstreks von 1969 also noch mehr oder weniger fraglos, d. h. ohne Veränderungen der entwickelten gewerkschaftspolitischen Linie, in die tradierte Politik der Gewerkschaftstïrhung eingebaut werden, in dem sich die formulierten Lohnforderungen an den gestiegenen Gewinnen orientierten, sich selbst auf die ron Wirtschaftsminister Schiller propagierte Soziale Symmetrie" (17) beziehen konnten, so entuickelt sich in der sich ausweitenden Orientierung der Arbeiterforderungen an ihrer Reproduktion der Widerspruch zur offiziellen bürgerlichen Gewerkschaftspolitik weitaus schärê. Dieser Widerspruch lafs sich in zweierlei Richtung fassen: Zum emen wenden sich die Streiks gegen die zur Reallohnstagnation bis hin zur Reallohnsenkung führende Politik der IG-Metall, sofern diese sich auf die gesamte Lohnhöhe bezieht. Zum anderen wird zunehmend durch die Streiks auch praktisch eine Politik der Gewerkschaft kritisiert, welche die Bedingungen der Anwendung der Arbeishlraft dem einzelnen Kapitalisten überlafit. Dies wird besonders beim Ford-Streik deutlich. Aber auch in anderen betrieblichen Streiks werden Forderungen gegen die Anwendung bestimmter Lohnsysteme, gegen Akkorderhöung etc. formuliert (18). In zwei Punkten also erweist sich die historisch herausgebilde-

17) Vgl. MiSF. Die Septemberstreik: 1969 a. a. O. S. 238 t. Zwar liegen der Streikbewegung atuh Erfahrungen von Arbeitintensivierung und Produktionssteigerung ohne Rucksicht auf die Arbeiter und ohne entsprechende Lohnerhöhung zugrunde. doch setzt sich diese Eriahrung noch nicht in spezificche Forderungen um.

18) Her eine vorläufge Aufitellung von Forderungen. welche sich aut Arbeitsbedingungen, die Reproduktion der Arbeiter. die Deklassierung der unteren Arbeiterschichten, Lohnsysteme etc. richten

25. 1. Hoesch-Rochrenwerke AG-Barop. Cnzufriedenheit mit Lohntindungsprinzip und Pramiensustem:

26. 1.-8. 3. Mannesmann/Huckingen. Verbesserung des Pramiensystems und der Arbeitsbedingungen:

4.-7. 5. Karmann Onabrück. 4 Wochen Betriebsurlaub für auslandiche Arbeiter (Portugiesen und Spamer streiken):

15.-16. 5. Heidelberger Schnellpresse Wiesloch. 6 Wochen statt bisher 4 Wochen zusammenhängenden Lrlaub (Spanier streiken):

19.-22. 5.. John Deere/Mannheim, zunächst Änderung der Akkordregelung, dann Lohnforderung:

10. 8.. Fernschapparatefabrik Philipp/Krefeld. Rücknahme der Erhöung der Tagesproduktion:

13.-20. 8., Pierburg AG/Neuss, Teuerungszulage. Aufhebung der Leichtlohngruppe 2 (vor allem ausländische Arbeiterinnen): 
te. auf die Gesamtlohnbewegung unter Beruksichtigung enkommenspolistiser Ge sichtspunkte hin orientierte Politik der lobletall her als wberholt: sie wird zun einen durch die inflationare Entwicklung unieflaufen. Zum anderen durch die Fa. higkeit des Kapitals. Mehratbeit im Produktionsprozeh durch die verschiedensten Formen der Intensivierung der Arbeit abzupressen. Dat herbei die drohende Ar. beitsplatzunsicherheit zusätzlich als disziplinierendes hittel der a tuspressung ron Mehrarbeit rom Kapital eingesetzt und. zeigt ebentalls det Zusammenhang con Arbeitsintensivierung und Entlassungen. Welche den Ausgangspunkt des Ford. Streiks bilden (19).

In unserem lezzten Aufsatz (20) waren wir zu dem Resultat gekommen. dar sich die Beschränkung der Lohnrate. die frbeitsplatzunsicherheit und die wachsen-

21. 8. Manchinenfabrik. F. Tacke Rheine. Vorgabezeitwerkurungen:

22. -27.8. Opel'Bochum. Teuerungsulage von 300 DV. din Tag mehr Lriab fur Sonderehichten. Wehnachtogeld auf Grundlage von 185 Stunden. bezahite Pauc ron 30 Minutin:

24. -30.8 . Ford holn. vgl. Fulnore 19 ,

24.- 29. 8. Philipp Aachen. Teurungzulage. Erhohung de Stundenohn Hegtall der Lohngruppe 2. Wegrall der Sametagsichicht:

24. 8. Vercinigte Schluseherke Solingen. 1 Duf. Senkung des fikords.

19) In der Hall. in wheher der Streik begann. wurde nach Angaben der Arbelrer trot?

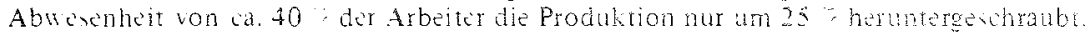
Zudem seien zu diexer Zeit Stopper an den Bandern ershienen. Es bostand alio de Befurchung. dat die Cincmehmensleitling die Entlasung der Arbeiter unter vorgechobenem Grund zum Anlaw einer weiteren Steigerung des arbeitstempon fur die Cbrig. geblicbenen ausnutzen wurde. In einem Flugblatt des IG-Metall Vertrabenskorpers wax schon Mitte August vermutet worden. dak .in Zukunit noch mehr Druck auf die betrof* fenen kollegen und Kolleginnen" ausgeubr werden würde. Ein ...Heter" des englischen Fordmandgement, hatte dis .. Lnverschamatheit" besessen. .au Grunden der Fostener. sparnis die Reduktion der ..Cberstunden aut ein Minimum . . bei gleichzeitiger Reduktion der Planstellon” zu fordern. Die DK.P-Zeitung. Der Prufstand spricht ron 57.4 Ent" lassungen im August mit dem vorgeschobenen Grund des unentschuldigten Fehlens. Sie geht daron aus. dats zum einen ein gezielter Personalabbau, zum anderen Disziplinierung der Belegschaft damit besbsichtigt sei. De Arbeiter mutsten demnach mit eniger Sicherheit annehmen. dats die Entlassungen auf ihrem Rucken ausgetragen wirden. So ist es kein Zufall. date ihre Solidariat mit den entlassenen Kollegen. Austosungsmoment des Streik: unmittelbar serknüpt war mit der Forderung nach Verminderung des Arbeitstempos. Insgesame nimmt der innere Zusammenhang der Forderungen solchen Vorstellungen jegliche Grundlage. Welche die Kampfinhalte von Streikbew egungen in qualitative, auf die Arbeitsbedingungen gerichtete. potentiell revolutionäre auf der cinen und quantitative, nur auf den Lohn gerichtete. das Sistem stabilisierende Forderungen auf der anderen Seite auseinanderdividieren wollen. Gerade für die unteren serbeiterschich. ter. Welche in den niedrigsten Lohngruppen eingestuft sind di: Arbeiterschichter also. welche im Fortgang der Akkumulation des BRD-Kapitals sich mehr und mehr mit ausländischen Arbeitern. mit Frauen. mit proletarisierten Bauem aus dem Lmkreis der croben Industriezentren auffüllen. aber auch jungen Arbeitern ist die Trennung eine leere Abstraktion. Die allgemeine Aiedrigkeit des Lohns, jetzt noch zusammergenommen mit den hohen Inflationsraten. erzwingt selbst weder Leistungssteigerung. Cberstunden. Akkordschinderei. Diese Erfahrung, zusammengenommen mit der an den Entlassungen demon. strierten Unsicherheit des Arbeitsplatzes, bilden die Grundlage der Forderungen des Ford-Streiks. In ihnen, vorgetragen vor allem von den turkischen Arbeitern. Wird ver sucht. den beschriebenen Zirkel zu durchbrechen. 
de Arbeitsbelastung als allgemeine Probleme der Arbeiterklasse in der BRD in starkerem Ilafe geltend machen. und daß sich daraus zunächst die Moglichkeit von Konilkten ergibi, die selbst wieder die mögliche Grundlage der Bildung von Klas. senbevulsein und damit der Vereinheitlichung der Arbeiter als Klasse gegenüber den Kapital bilden. Damit seien die zukünftigen Probleme für die gewerkschaftliche Interessenvertretung der Arbeiter gestellt. die zugleich die politische Artikulierung de: Arbeiterklasse gegenüber dem Kapital und dem Staat befördern werden. Gleichzeitig waren wir davon ausgegangen. daf ein solcher Prozes angesichts der Zusammensezung der Arbeiterklasse in der BRD (ausländische Arbeiter und Frauen bilden einen grofen Anteil der am niedrigsten bezahlten und von der Arbeitsintensivierung am schwersten betroffenen Schichten der Lohnarbeiter in der BRD) und im Hinblick auf den gegenwärtigen Zustand der Gewerkschaftsorganisation in sich selbst widerspruchlich und ungleichmärig verlaufen dürfte.

vun fällt an der Streikbewegung 1973 folgendes ins Auge: Im Unterschied zu 1969. wo die Streikinitiative vor allem von der Schicht der Facharbeiter ausging (.1), übernehmen in diesem Jahr die unteren Schichten der Arbeiterklasse in vielen Betrieben die Streikinitiative. Dies gilt schon für den Hoesch-Streik, entwickelt sich dann abe:weiter als Streikinitiative und zunehmende Kampfbereitschaft gerude der ausländischen Arbeiterinnen und Arbeiter bei Karmann/Osnabrück, John Deere Hannheim. Hella Lippstadt. Pierburg/Neuß und Ford/Koln. Die Initiative Funktion dieser Schichten prägt gleichzeitig die neue Militanz der Kämpfe. Die Militanz und die Formen, in denen die ausländischen Arbeiterinnen und Arbeiter die Sireiks führen. bildet andererseits partiell die Grundlage von Konflikten mit deutschen Arbeitern. welche vom Kapital - bei Ford ganz offensichtlich mit Unterstüzung des Betriebsrats - zur Spaltung zwischen deutschen und ausländischen Arbeitern und zum brutalen Polizeieinsatz gerade gegen die ausländischen Arbeiter ausgentitzt wird. Der Gegensatz zwischen traditioneller, sozialdemokratisch orientierter Gewerkschaftspolitik und Arbeiterinteressen wird in dieser Streikbewegung am vehementesten von den ausländischen Arbeitern formuliert. Es zeigt sich hier ein weiteres entscheidendes Problem der Gewerkschaft in der BR.D: die mangelnde Orientierung der gewerkschaftlichen Interessenvertre tung auf die Lebens- und Arbeitsbedingungen der unteren Schichten der Lohnarbeiter. der ausländischen Arbeiter und der Frauen. sowie die Strategie des Kapitals, diese Schwäche für sich zur Spaltung der Arbeiterklasse auszunutzen. Die beschämendste Niederlage der Gewerkschaft in diesen Kämpfen stellt die Kooperation von Unternehmensleitung, Teilen des Betriebsrats und der Vertrauensleute und Polizei gegen die türkischen und Teile der deutschen Arbeiter bei Ford dar.

Im Prozefo der Entwicklung der Klassenkämpfe aufgrund der Bedingungen, denen die Arbeiter im Akkumulationsprozef des Kapitals gegenwärtig unterliegen, sind also Moglichkeiten für das Kapital enthalten, von vornherein zu versuchen, die Vereinhellichung der Arbeiter als die wesentliche Aufgabe jeder Klassenbewegung zu hintertreiben. Diese Möglichkeit basiert auf der vom Kapital teils bewußt produ-

21) MVSF (Hrsg.). Die Septemberstreiks 1969. a. a. O. .. Lnter berufsspezifischen Gesichtspunkten sind in der Wehrheit der Betriebe qualifizierte Arbeitergruppen die tragenden und treibenden Kräte des Streiks." (Ebd.. S. 240) 
zierten tendenziellen Teilung zwischen deutschen und ausländischen Arbeitern in untere Schichten einerseits und Facharbeiter andererseits, vor allem in den grofen Industriebetrieben. besonders ausgeprägt wiederum in der Automobilindustrie (BMW. Ford. Daimler Benz). Dies führt einmal dazu. dafo die Intensivierung der Arbeit als Streikgegenstand von vornherein schwer verallgememert werden kann. (Dafi es sich dabei nicht um eine absolut zu setzende Schwierigkeit handelt. haben wir im Aufsatz ..Zum Zusammenhang von Kapitalakkumulation. Veränderungen in den Formen der Profitproduktion und der Rolle neuerer Entlohnungsmethoden in der BRD" (PROKLA Nr. 4 und 5) zu zeigen versucht: nämlich Intensivierung und Dequalifikation betreffen zunehmend auch traditionelle Arbeiterschichten. Sun kommt der Gewerkschaft an sich die Aufgabe zu. als Organisation die Konkurrenz zwischen den Arbeitern. d.h. auch zwischen den verschiedenen Arbeiterschichte? abzubauen und aufzuheben, sofern sich in dieser Konkurrenz auch untersiniediche Bedingungen der Arbeiter reflektieren. Wo Forderungen nicht aus sich heraus ron vornherein von allen Arbeitersihichten gleichmäfig vettreten werden. ware es eben gerade Aufgabe der Gewerkschaft. über die Organisation eine Moglichkeit allgemel. ner Solidaritat herzustellen. Gerade dies geschieht aber. wie die Strekis gezeigt haben. nicht oder nur in Ausnahmefällen seltens besonders bewufter betrieblicher Kader. Hiermit kommen wir auf ein weiteres Problem der gegenwärtigen Schwähe der Gewerkschaft: gerade fur die IG-Metall ist typisch. daf sie sich. auch in ihron betrieblichen Organisationen der Vertrauensleute, primär auf die Facharbeiterschichten stüzt und vor allem deren Interessen gegenuber dem Kapital vertrit. die ausländischen Arbeiter demgegenuber in der Gewerkschaft weitgehend isolier sind. (Wieweit dies allgemein für die unteren Schichten der Arbeiter und ihre Position innerhalb der gewerkschaftlichen Interessenvertretung gilt. wäre noch zu unterss. chen.)

\section{Grenzen der gegenwärtigen Politik betrieblicher Gewerkschaftskader werten sichtbar}

Es ist wohl kein Zufall, daf die Grenzen der Politik auch linker betrieblicher Gewerkschaftskader in einer Streikbewegung offenbar werden. in der die unteren Schichten der Arbeiter of die Initiatoren und Hauptträger des Srreiks sind. 1969 war demgegenuber die Streikbewegung von den betrieblichen Vertretungsorganen z. T. getragen, z. T. mehr oder weniger widerspruchslos aufgenommen worden (2)). Typisch ist hier wieder der Hoesch-Streik. wo 1969 Betriebsräte und Vertrauensleu. te den Streik initiiert hatten, 1973 dagegen der Vertrauenskörper nicht in der Lage ist, eine von den Arbeitern an der Feineisenstrake ausgehende, von ihm nicht initi. ierte Streikbewegung zu organisieren und zu einem Erfolg zu bringen. Bei Ford. einer Hochburg der Gewerkschaftslinken in den 60er Jahren (dort war ubrigens auch versucht worden eine betriebsnahe Tarifpolitik. Kontrolle der Bandgeschwindigkeiten usw. durch einen gewerkschaftlich kontrohlierten Betriebsrat durchzuset. zen), führen u. a. die nur innerhalb der betrieblichen Institutionen des Berriebsrats 22) Vgl. ebd. S. $25 v-253$ 
und Vertrauenskorpers ausgefochtenen Fraktionskämpfe zwischen rechtem BR und Gewerkschaftslinken. die sich auf personelle Stellenwechsel beziehen, zur weitgehenden Lahmung dieser Institutionen zu Beginn des Streiks. zu ihrer durchgehenden Distanzierung und teilweisen Bekämpfung der selbständigen Streikleitung, die selbst weder Resultat des Zustands der betrieblichen Interessenvertre tungsorgane ist. Hier wird auch deutlich. wie der rechte Betriebsrat durch seine Art der Verhandlung selbst die Spaltung zwischen deutschen und ausländischen Arbeitern forciert, indem er die Verhandlung dann für beendet erklärt. als die Forderung nach Lohnerhöhung, die von allen vertreten wurde. partiell durchgesetz.t ist. die be sonderen Forderungen der ausländischen Arbeiter (Lrlaub. Arbeitsbedingungen) dagegen nicht weiterzurerhandeln bereit ist.

Ein weiteres Moment der gegenwärtigen Grenzen der Politik auch linker betrieblicher Gewerkschaftskader. wird in der Schwäche dieser Kader sichtbar, sobald sich die Gewerkschaftszentrale von dem Streik distanziert. Im Septemberstre ik hatten die betrieblichen Kader den Ruckenwind einer verhandlungsbereiten Gewerkschaftsfuhrung. vor allem in der IG-Netall. (Die besondere Situation im Bergbau können wir hier nicht behandehn). Dies gilt 1973 nicht mehr. Fehlt also einerseits häufig die wirkliche Verbindung zwischen der Politik auch linker Gewerkschafter im Betrieb mit den unteren Schichten der Arbeiter, welche den Streik tragen, so entspricht dem auf der anderen Seite, dafi sie z. T. auch nicht selbstandig und selbstbew ufit den Konflikt mit der IG-Metallspitze auszutragen in der Lage sind. (Auf die Grinde. welche fur diese Erscheinung in der institutionellen Organisation des Vertrauenskorpers in Verhältnis zu Betriebsrat. Lnternehmensleitung und Gewerkschaftsapparat zu suchen sind. soll in einem später zu veröffentlichenden Aufsatz eingegangen werden.)

Das Problem kann noch an folgender Entwicklung verdeutlicht werden: Im April und Mai kommt es zu einer breiten Formulierung von Forderungen aus den Vertrauenskorpern. aber auch den örtlichen Vertreterversammlungen an die Gewerkschaftsfuhrung. die sich auf eine Entfristung der Tarifverträge richten. Doch dieser Versuch. aus den Betrieben heraus Einflurs auf die Gesamtlohnbewegung zu gewinnen, der Zersplitterungstaktik des Kapitals entgegenzuwirken, bleibt erfolglos. Die Gewerkschaftsfihrung macht nicht den Versuch. durch gewerkschaftlich initiierte betriebliche Streiks die nötige Lnterstiitzung für die Verhandlungen sich zu sichern. Sie verhandelt ohne Erfolg auf Spitzenebene, nicht einmal die große Tarifkommission wurde zu den Verhandlungen hinzugezogen. Während die betrieblichen und ortlichen Gewerkschaftsfunktionäre ihre Forderungen gegenüber der IG-Metallspitze formulieren, beginnen in den Betrieben die Streiks um Teuerungszulage. Sie richten sich unmittelbar an den einzelnen Lnternehmer, die Arbeiter kämpfen da, wo sie kämpfen können, auf Betriebsebene. Die Forderungen richten sich nicht an die Gewerkschaft. Es fällt auf. wie in dieser Phase die Aktionen betrieblicher Gewerkschaftskader und Aktionen der Arbeiter auseinanderzulaufen beginnen. Mögen nun auch die Gründe, aus denen Vertreterversammlungen und Vertrauenskörper ihre Appelle an die Gewerkschaftsführung richten. unterschiedlich sein. mag bei den einen die Vorstellung bestimmend gewesen sein. die Gewerkschaft solle durch ihre Initiative eine Streikbewegung verhindern. mögen andere davon ausgegangen sein. 
die Gewerkschaft müsse dazu gezwungen werden, die Kamplbercitschalt in den Betrieben auszunutzen, um ein besseres allgcmeine's Ergebnis zu crielen; in jedem Fall fällt auf, dak die Streiks sich nicht auf eine Unterstütung der Spiczengespräche richten, sondern auf die Auseinandersetzung mit dem Kapital in Betrieb. Darin reflektiert sich die Erfahrung der Arbeiter von dem Ergebnis der Tarifverhandlung, der Nutzlosigkeit der Streikbereitschaft der Arbeiter in Bezug auf das Handeln der Gewerkschaftsführung. Dieses Nebeneinanderherlaufen entwickelt sich dann bei Ford zum eklatantesten Widerspruch. Ford war der Betrieb, von dem aus mehrmals sowohl über den Vertrauenskorper, als auch über Belegschaftsversammlungen, als auch über die Ortsverwaltung Appelle an die IG-Metallführung gegangen waren.

Aber selbst in Betrieben, wo selbstbewuk te linke Betriebsräte den Streik ohne Legitimierung der Gewerkschaftsführung führen, kommt es zu Niedierlagen. So etwa bei AEG-Küppersbusch. Hier läßt sich einfach der Konzern, dem der Betrieb angehört, durch den Streik eines Einzelbetriebs nicht zwingen. Eine Solidarisierung aus anderen Betrieben des Konzerns bleibt aus. Die Auffassung, dak bei einem der Belegschaft verpflichte ten Betriebsrat oder bzw. und bei einem starken, unabhängigen VK innerhalb einer relativ günstigen ökonomischen Situation der Kanıf gegen den einzelnen Kapitalisten erfolgreich geführt werden könne, muk aufgrund der aktuellen Kämpfe neu überprüft werden.

\section{Zusammenfassende Ü berlegungen}

Wenn es eine politisch hewußte Strategie des Kupitals gibt, so immer die der Schwächung der Arbeiterklasse durch Zersplitterung und Spaltung. Der Entwicklungsgrad des Klassenkampfs von oben ist dabei nur Reflex der verschlechterten Bedingungen der Reproduktion und Akkumulation des Kapitals (23). Dabei sei noch einmal daran erinnert, daß sich zwar im Vergleich zu dem rasanten Aufschwung nach der Krise 1967, in welchen die Septemberstreiks 1969 fallen, die Bedingungen der Profitproduktion fur das Kapital im Jahre 1973 verschlechtert haben, daß sich aber trotzdem die Arbeiterklasse in diesem $\mathbf{J a h r}$ aufgrund des nicht vorhandenen aktuellen Drucks einer industriellen Reservearmee und der Auftragslage des Kapitals in einer relativ starken ökonomischen Position gegenüber dem Kapital befindet. Diese relative ökonomische Stärke versucht das Kapital jetzt aber politisch zu unterlaufen. Zum einen durch die Strategie, das Zustandekommen einer

23) Wir haben im letzten Heft der PROKLA (Nr. 8/9) in Auscinandersetzung mit der RGOPolitik der KPD/AO-Gruppe gezeigt, dałs der stattfindende Prozets der Enttäuschung der Arbeiter über das Nich tfunktionieren der Gewerkschaft als Kampfïnstrument notwendige Voraussetzung überhaupt für die Entwicklung cines Klassenbewulitseins unter den gegenwärtigen Verhältnissen ist. Der Lernprozef, der hier gemacht werden mufs, zielt auf dje Erkenntnis, dab die Gewerkschaftsorganisation als Kampfinstrument der Arbeiter nur dann funktionieren kann, wenn sie von den Arbeitern über die Austragung von Konflikten innerhalb derselben auch wirklich zu einem solchen gemacht wird. Diese Erkenntnis bedeutet auch die Überwindung einer Schranke im Bewuf3tsein der Arbeiter selbst, nämlich der Vorstellung, per Delegation der Interessenwahrnehmung an die Gewerkschaftsorganisation oder die Betriebsräte sei ihre gesellschaftliche Stellung zu verteidigen. 
geschlossenen spontanen Streikbewegung zu verhindern. Dazu hat ihm der niedrige Lohnabschluß der IG-Metall in den Einzelbetrieben den nötigen Spielraum für jeweilige Lohnerhöhungen gelassen. Zum andern aber auch durch den Einsatz von Polizei in einem seit lantes nicht mehr erlebten Ausmaß. Während der Streiks wird spätestens dann gege: wi fiberter ein Polizeieinsatz inszeniert, wenn das Kapital durch Weiterführung der Streiks ökonomisch zu Zugeständnissen gezwungen gewesen wäre.

Wieweit eine solche Strategie des Kapitals Erfolg hat, hängt andererseits von dem historisch entwickelten Charakter der Gewerkschaftsorganisation und der Klassenkämpfe ab. In den Niederlagen der Streikbewegung 1973 reflektiert sich demnach die Schwäche der Gewerkschaftsorganisation als Klassenorganisation. Entscheidende Einbruchsstellen für die Strategie des Kapitals sind: Die historisch herausgebildete Beschränkung der Gewerkschaft auf die Gesamtlohnbewegung, d. h. die Ausgrenzung des Verhältnisses zwischen Lohnarbeit und Kapital im Produktionsprozeß aus dem Bereich ihrer Interessenwahrnehmung und -repräsentation; die ebenfalls historisch begründete Festlegung der Gewerkschaftsführung, aber auch größerer Teile der Betriebsräte und Vertrauensleute auf die Unterstützung der Politik der SPD; die politisch durchgesetzte Trennung zwischen betrieblicher Interessenvertretung im Betriebsrat und der Gewerkschaft (BetrVG); die entsprechende Festlegung des Betriebsrats auf die Friedenspflicht, d. h. die institutionell un rechtlich verhinderte bzw. eingeschränkte Kampffähigkeit der Gewerkschaften auf Betriebsebene; die außerordentlich schwache Stellung der betrieblichen Gewerkschaftsorgane (Vertrauensleute) gegenüber dem Gewerkschaftsapparat, ihr mangelnder Einfluß auf die tarifpolitischen Entscheidungen, aber auch ihre fehlende Verhandlungsmöglichkeit mit dem Unternehmer; die innere Schwäche der Vertrauenskörper selbst, insofern in ihnen oft nur die deutschen Facharbeiter, nicht aber die unteren Schichten der Arbeiter repräsentiert sind; die Abhängigkeit der Vertrauensleute vom Betriebsrat; das Fehlen einer inhaltlich entwickelten Strategie gegen das Kapital auch in Teilen der Gewerkschaftslinken. Der starke organisatorische Apparat verdeckt nicht mehr den tatsächlichen Zustand der Zersplitterung der Klasse. Der Inhalt der Gewerkschaftseinheit als Einheit der Arbeiterklasse gegen das Kapital wird ins Gegenteil verkehrt, wenn sich die Gewerkschaftsführung auf die Einheit der Gewerkschaft als Bedingung der Stärke der Arbeiter gegen die spontanen Streikbewegungen beruft; wenn rechte Betriebsräte, wie bei Ford geschehen, sich weigern, die Forderungen der Arbeiter gegenüber der Unternehmensleitung zu vertreten mit der Begründung, dies laufe auf Betriebssyndikalismus hinaus; wenn sich demnach die Gewerkschaft auf rechte Betriebsräte stützen muß, um ihren Anspruch, die Einheit der Arbeiter zu repräsentieren, aufrechtzuerhalten. Dies in einer Situation, wo diese einheitliche Organisation der Arbeiter wenig produktive Potenzen zur Entwicklung der Klassenkämpfe freizusetzen vermag, wo innerhalb der Gewerkschaft versucht wird, die Kritik an der offiziellen Gewerkschaftspolitik mit dem Verdikt des gewerkschaftsschädigenden bzw. spalterischen Verhaltens zu unterdrücken; wo die inneren Organe der Gewerkschaft, soweit sie über den Betrieb hinausgehen, wenig Raum lassen für die Auseinandersetzung um eine Strategie der Arbeiter gegen das Kapital; wo die linken Kräfte innerhalb der Gewerkschaft nur schwer zu einer über 
den Betrieb hinausgehenden Kooperation kommen können, sondern auf eine betriebsbornierte Politik zurückgeworfen zu werden drohen; wo auf der anderen Seite gerade die Beschränkung der linken Gewerkschaftskader auf einen betriebsinternen Spielraum selbst die Grenzen einer solchen auf den Betrieb beschränkten Politik sichtbar machen, nämlich dort, wo sich auf der Ebene des Einzelbetriebs das Kapital nicht zwingen läßt. Indem die Gewerkschaft als überbetriebliche Organisation nicht die Bewegung solcher Avantgardebetriebe aufnimmt, wird der Erfolg soicher Kämpfe auf der Ebene der Einzelbetriebe in Frage gestellt. Andererseits können die Kampfpotenzen solcher Betriebe auch nicht zu Machtpositionen der Arbeiterklasse gegen das Kapital, vermittelt durch die Gewerkschaftsorganisation, entwickelt werden.

Daß es zu keiner geschlossenen Streikwelle kommt, sondern die Kämpfe sich zersplittern, dafür gibt es noch einen weiteren Grund. In der gegebenen Situation des Drucks auf die Lohnrate und des Versuchs des Kapitals, durch neue Lohnsysteme, Veränderung des Akkords, Rationalisierungsmaßnahmen und Entlassungen die Profite zu erhöhen, zeigt sich die Unfähigkeit des gewerkschaftlichen Kampfs gegen die vom Kapital innerhalb der Betriebe je spezifisch gesetzten Lohn- und Arbeitsbedingungen wirksam anzugehen; was dann zu spontanen Streiks führt, die sich durch die Forderung nach Teuerungszulage als der gewerkschaftlich schon anerkannten Forderung zu legitimieren und durchzusetzen suchen. Oft sind es eben solche innerbetrieblichen Konflikte, die den Streik auslösen; wobei die Forderung nach Teuerungszulage die einfachste Möglichkeit für die Belegschaften bildet, den Streik innerhalb des Betriebs zu verallgemeinern (exemplarisch bei John Deere). Dies zeigt aber nur, daß die Notwendigkeit zu solchen einzelbetrieblichen Kämpfen existiert, die Unfähigkeit aber, diese Kämpfe gewerkschaftlich zu organisieren, gleichzeitig zum Resultat hat, daß auch keine geschlossene Streikbewegung zustande kommt. Dies zeigt, daß die Alternative nicht die zwischen zentralen und betrieblichen Kämpfen ist, sondern daß verallgemeinerte, zentrale Kämpfe nur geführt werden können, wenn auch auf Betriebsebene die Auseinandersetzung mit dem Kapital um die je spezifischen Arbeits- und Entlohnungsbedingungen geführt werden kann.

So desolat sich der Zustand der Gewerkschaft innerhalb der Streikbewegung des Jahres 1973 zeigt, so kämpferisch sind oft auf der anderen Seite die betrieblichen Streiks. Während die Gewerkschaftslinke jahrelang davon ausging, ein wesentliches Problem gewerkschaftlicher Kampfstärke bildete die mangelnde ,Mobilisierungs der Belegschaften, sieht sie sich jetzt zunehmend vor das Problem gestellt, daß in Betrieben, wo gestreikt wird, die Gewerkschaft nicht in der Lage und nicht gewillt ist, diese Kampfbereitschaft in eine Machtposition der Arbeiter gegen das Kapital umzusetzen. So entscheidend wichtig die gegenwärtige Streik be wegung für die Wiederentwicklung der Kampffähigkeit und des Klassen bewußtseins der Arbeiter ist, so steht sie doch vor dem Dilemma, daß die Waffe der gewerkschaftlich organisierten Einheit der Arbeiter gegen das Kapital stumpf ist. Andererseits liegt die ganze Stärke und der Fortschritt dieser Klassenbewegung darin, daß sie das Problem der Gewerkschaftseinheit als Klasseneinheit erstmals klar und deutlich auf die Tagesordnung setzt, nicht nur für vereinzelte, klassenbewußte Arbeiter und Gewerkschaftskader, sondern für größere Teile der Arbeiter und der betrieblichen Gewerkschafts- 
organe. Die Niederlagen bilden selbst die Voraussetzung für breiter getragene Auseinandersetzungen innerhalb der Gewerkschaft um deren Politik. Hier kann die ihres Inhalts entleerte Form der Einheitsgewerkschaft, einer Gewerkschaftsorganisation. die zum einen alle Schichten der Arbeiter in sich vereinigt, zum anderen nicht politisch in Richtungsgewerkschaften gespalten ist, wieder zur produktiven Potenz der Klassenauseinandersetzung werden. Vermittelt über die Form der Einheitsgewerkschaft wird notwendigerweise unter dem Druck der gegenwärtigen Niederlagen dic Auseinandersetzung um die Kampffähigkeit und bereitschaft der Gesamtorgansation in diese hineingetragen. Dies kann auf allen Ebenen der Organisation geschehen: In der Auseinandersetzung zwischen rechtssozialdemokratischen Betriebsräten und Vertrauensleuten mit der Gewerkschaftslinken und den Arbeitern im Betrieb. im Versuch der Entwicklung von Widerstand gegen die offizielle Gewerkschaftspolitik durch einzelne Ortsverwaltungen: aber auch, wie die IG-Metall in Nordbaden Nordwürtemberg zeigt, durch eine wie immer auch motivierte Ausrichtung der Tarifpolitik auf das Problem der Arbeitsbedingungen in den Betrieben innerhalb einer Region. Gerade bei dieser neuen Entwicklung in Nordbaden/Nordwürtemberg ist zu lragen, wieweit die Tatsache, dak der Gewerkschaftsapparat selbst den bisher nicht zur Kenntnis genommenen Ausbeutungsprozek im Betrieb zum Gegenstand gewerkschaftlichen Kampfes macht, für die Zukunft Bewegungen freisetzt und legiimiert, die der gegenwärtigen Schwäche der gewerkschaftlichen Organ'sation als Kampforganisation im Betrieb entgegenwirken können. Denn die Herrschaft des Kapitals uber den Produktionsprozel impliziert, dak das Kapital versuchen wird. jede durch allgemeine Tarifauseinandersetzungen durchgese tzten Zugeständnisse auf Betricbsebene wieder rückgängig zu machen, wenn sich dem nicht kampffähige Organisationen der Arbeiter im Betrieb entgegenstellen: eine Erfahrung übrigens, die sich z. B. in Italien in der Delegiertenbewegung reflektiert (24).

Aber auch im Propagieren des Konzepts der betriebsnahen Tarifpolitik reflektiert sich. wie immer auch noch unfertig, das Begreifen der Notwendigkeit. die Kamptbereitschatt und -fähigkeit der Arbeiter auf Betriebsebene für die Politik der Gewerkschaf tsorganisation als vorantreibendes Moment nutzbar zu machen, auf der anderen Seite die gewerkschaftliche Kampffähigkeit auf Betriebsebene überhaupt erst wieder herzustellen. Dies wird besonders dann deutlich, wemn der Zusamnenhang zwischen dem Postulat nach betriebsnaher Tarifpolitik und Stärkung der Stellung der Vertrauensleute innerhalb der Organisation gesehen wird. Wenigstens übersehen die Kritiken, die von der Linken an dieser Forderung nach betriebsnaher Tarifpolitik geübt werden, den tatsächlichen Zustand der Zersplitterung, welcher durch die getrennte Interessenorganisation in Betriebsrat und Gewerkschaft gesetzt ist. Dats solche Konzepte sich noch weitgehend auf der Ebene organisatorischer Vorstellungen bewegen, sich erst allmählich mit Inhalten einer Klassenpolitik gegen das Kapital auffülen werden, sagt nur etwas uber ihre Unfertigkeit angesichts der Tatsache, daß die Probleme der Klasseneinheit, wie zu zeigen versucht wurde, sich konkret auch erst über Kämpfe, Erfolge und Niederlagen der Klassenbewegung herausarbeiten. In einem späteren Aufsatz soll auf diese in der Gewerkschaftslinken

24) Vgl. dazu das demnächst in Merve-Verlag erseheinende Buch über die italienische Gewerkschaftsbewegung, insbesondere den dort veroffentlichten Autsatz von Garavini. 
sich en twickelnden Konzeptionen und Strategien noch genauer unter den Fragestellungen, die wir an der Analyse dieser Streikbewegung gewonnen haben, cingegangen werden. Soviel sei noch gesagt: Solange die Vernachlässigung des Problems der Kanpffahigkeit der Gewerkschaft auf Betriebsebene nicht durch die Erfahrungen selbst als entscheidende Schwäche der Gewerkschafi sichthar wurde, kommicn vom Gewerkschaftsapparat Konzeptionen in dieser Richtung als Siorfaktoren der einkommenspolitischen Linie der Gewerkschaftsführung abgekappt und unterdrückt werden. Je mehr dagegen die Strategie des Kapitals offen sichtbar wird (als Versuch nämlich, durch die Beschränkung der Gewerkschaft auf national ausgehandelte Tarife und die damit geförderte Entfremdung von den Mitgliedern der Gewerkschatt jeden Einfluts auf die wirklichen Lebensbedingungen der Arbeiter zu nelmen), desto mehr sind gerade auch die unteren Gewerkschaftsfunktionäre dazu gezwungen. sich fur eine Wiederherstellung der Kampffahigkeit der Gewerkschafi aul allen Ebenen und eine innere Demokratisicrung der Entscheidungsprozesse einzuserzen. Die wesentliche Machtbasis, auf welche sie sich dabei stutzen mussen, sind die Kämpfe der Arbeiter in den Betrieben selbst.

Zusammengefarkt handelt es sich darum, dark die Klasseneinheit sich nich organisatorisch im Gewerkschaftsapparat darstellen kann, sondem dab diese Linheit wesentlich Resultat des Klassenkampfs zwischen Lohnarbeit und Kapital sein kann, von den Arbeitern selbst als ihr Problem erfahren und entwickell werden muks. Dabei kann die konkrete historische Verfassung der Gewerkschafisorganisaiion dicsen Prozefs befordern oder henmen. Das gegenwätig entscheidende Hemmnis der Entwicklung bildet die Bindung zwischen SPD und Gewerkschaft, sowie dic Machtposition rechter SPD-Funktionäre innerhalb der Gewerkschaft. Für die weitere A nalyse der Klassenentwicklung in der BRD gilt es also, die Ansatzpunkte und llebel aufıuzeigen, über welche die gegenwärtigen Erfahrungen von Niederlagen umgese $z$ t werden können in Lermprozesse der Arbeiter und der Organisation selbst, Lernprozesse, in denen der Widerspruch zwischen bestehender bürgerlicher Politik und notwendiger Klassenpolitik in der Gewerkschaft ausgetragen wird und durch die die Gewerkschaft wieder fähig gemacht wird, die Aufgaben der Klassenbewegung in Kämpfen mit dem Kapital wahrzunehmen.

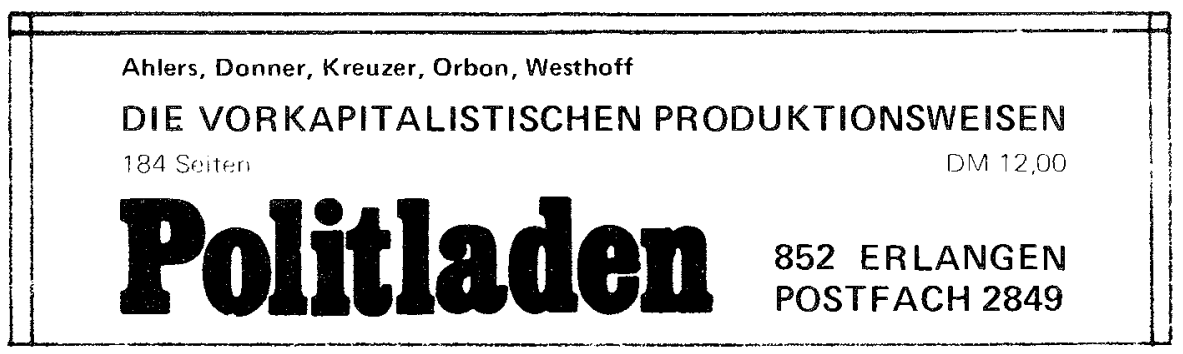

